\title{
A PHARMACOLOGICAL FRAMEWORK FOR INTEGRATING TREATING THE HOST, REPUPOSING AND THE DAMAGE RESPONSE FRAMEWORK IN COVID19 DISEASE
}

\author{
Richard Head ${ }^{1}$ and Jennifer Martin ${ }^{2}$ \\ ${ }^{1}$ University of South Australia \\ ${ }^{2}$ The University of Newcastle
}

July 6, 2020

\begin{abstract}
Historically in a pandemic the focus has been upon identifying agents that would eliminate the invading species with as little damage as possible to the host. Recently discussion has moved, particularly with COVID-19, from antiviral therapy to delineating the treatment of the host from treatment. With the latter, the approach brings together three concepts; treating the host, the damage response framework and therapeutic repurposing. The integration of these three areas play heavily to the traditional strength of pharmaceuticals in providing a period of stabilization prior to the introduction of subsequent interventions to permit time for the development of novel anti-viral drugs and vaccines. In integrating approaches to repurposing, host treatment and damage response we identified three key properties that a potentially effective repurposed drug must posses by way of a framework. There must be homology with the pathogenesis of the disease, ideally targeted to the conserved pathophysiological outcomes of the vial attack, have a defined locus within the spectrum from prevention to severe disease and finally draw upon the historical dose and safety experience of the repurposed drug. By way of illustration we have mapped therapeutic agents that impact upon the renin angiotensin system using this approach. Collectively this type of analysis reveals the importance of existing data (repurposed information and administrative observational data) and the details of the known pathophysiology of the viral attack are in approaches to treating the host; and with COVID-19 the significance of a pre-existing RAS-mediated inflammatory disposition.
\end{abstract}

\section{MAIN MANUSCRIPT}

\section{Introduction.}

Recently Sanders et al. (1) have highlighted both the fact that no therapies for treating COVID-19 were available at their time of writing and yet the need to have high quality evidence readily available in a pandemic. In a solution to this problem, we note there exists the potential confluence of two approaches that may prove beneficial in this pandemic setting. The first seeking agents that treat the host, not necessarily the virus, and building on the pioneering studies of David Fedson in this field (2-5); the second, the concept of repurposing existing therapeutics for new indications. Both concepts are enshrined in pharmacological principles.

In this Review we have below explored these concepts and provide a potential path for efficient evaluation of candidate drugs using inhibitors of the Renin Angiotensin System (RAS) as an example. 


\section{Treating the host.}

One of the key values of therapeutics is their potential in stabilizing patients prior to subsequent interventions or their use as adjuncts to those interventions. The settings where this may occur are varied and include response to traumatic injury to control damage, treat infection and alleviate pain. Other notable examples include the development of anaesthesiology and sedation to enable the performance of surgery and complex procedures, as well the development of non-depolarising neuromuscular-blocking drugs as adjuncts to those surgical procedures and of adjunctive chemotherapy to improve quality of life and survival time. Of particular interest in this pandemic context has been the centuries old development of agents to promote survival with infections. Historically the focus was upon identifying agents that would kill the invading species and not damage the host. In more recent times the focus has moved to delineating the treatment of the host from treatment and elimination of the infective agent.

In 2016, Fedson highlighted the treating of the host concept, based upon an understanding that a beneficial clinical phenotype has been observed with a treatment and this can be employed for the purpose of treating the host (3). This was done in the context of emerging virus diseases, and more recently in the context of severe COVID-19 infection (5). Consistent with this view Yan et al. (6) drew attention to the need for agents to alleviate H5N1 virus-induced lung injury due to the limited strategies for treating influenza virus infection.

The concept of treating the host is entirely complementary to opportunities arising in the area of drug repurposing that we have highlighted recently (7). In the case of COVID-19 disease this would mean identifying drugs that are focused, not on the elimination of the virus, but upon the survival of the patient with severe COVID-19 disease. This includes drugs approved for other indications that have the beneficial phenotype for treating severe COVID-19 disease. The fundamental advantages of this approach are; firstly, to buy needed time for the concurrent development of anti-viral or vaccines, secondly to provide health authorities a treatment insurance as they begin to release the public from social isolation. We have highlighted this key aspect recently in the context of Covid-19 disease (8). However this very obvious but distinct suite of solutions to reduce the morbidity and mortality from COVID has the potential risk of cutting across fixed interests and previously effective methods of succeeding in grant funding rounds, including silos of 'ology disciplines, and pressures from news cycles and campaigning for ineffective policies.

What is needed is the appropriate framework for treating the host, a strategy for positioning this approach, and then implementation.

\section{A framework for treating the host with repurposed drugs.}

Treating the host in COVID-19 brings together two key pharmacological principals. The first involves the understanding of how a proposed therapeutic agent integrates within the pathogenic response. This approach thus involves understanding initially the integration of the host into the consequences of viral pathogenesis. This relationship has been well described for microbial pathogenesis in the Damage-Response Framework (DRF) in infectious diseases (9). The damaging host factors described in this microbial model, namely cytokine release (storm), chemical mediators signalling, oxygen radical production and immune complexes leading to aberrant inflammation are not specific and in fact apply equally to viruses such as influenza and the current COVID-19 disease.

The second principal involves the appropriate use of therapeutics approved for one indication but used effectively in a different setting within the DRF. Approaches used for drug repurposing, the challenges and ways in which these challenges may be tackled have been described recently in detail (10). The application to COVID-19 involves identification of drugs that in a new indication, in the correct dosage for the new disease have a substantial impact on the consequences of infection in the host. 
A logical framework based on the above considerations for identifying repurposed drugs for treatment of the host would potentially include:

- Pathogenesis . Homology with the novel action of the repurposed drug and the molecular events that drive the consequences of viral pathogenesis in COVID-19 disease.

- Target homology of COVID-19 and therapeutic agents.Ideally the repurposed drug should target the pathophysiology of COVID-19 that is tightly linked to the conserved mutational properties of the virus and its class.

- Prevention to treatment. An understanding of how the repurposed drug provides benefit in treating the host across the cascade from prevention to the life-threatening consequences of other viral pathogenesis in COVID-19 disease.

- Dose and safety. Ideally an application of the repurposed drug either within the approved dose ranges for its initial indications for which the pharmacokinetics and toxicology are documented as part of the registration for the drug's initial indication, or

- A new application of an already registered drug in another indication, using doses based on pharmacological and physiological attributes of the new disease and the specific chosen effect of the drug at different dosages and/or plasma concentrations.

Using similar deduction, in 2020 Fedson et al. proposed clinical trials should be undertaken with combinations of the RAS agents angiotensin receptor blockers (ARB) and statins in patients with severe COVID-19 infection (5). His proposal provides an excellent opportunity to use our framework in evaluating repurposed RAS drugs for use in COVID-19. In the following example we focused upon drugs targeted to the RAS, but the procedure would equally apply to statins either alone or in combination with ARBs.

Exploration of the treating the host concept, using repurposed agents acting on the renin angiotensin system - ATC C09.

From the preceding discussion, a framework for treating the host and repurposing can be employed to explore the eligibility of drugs that influence the renin angiotensin system as candidates for clinical trials. In particular to identify what is known about these agents in regard to:

1) Pathogenesis 2) Target homology of COVID-19 3) Prevention/ Treatment and 4) Dose-Response curve in relation to safety.

1. Pathogenesis. To treat the host requires an understanding of the physiology of this response to that condition (in this case COVID). In addition, knowledge is needed of chemical, structural and immunological homology with the novel action of the repurposed drug and the molecular events that drive the consequences of viral pathogenesis in COVID-19 disease. As the main causes of death in COVID are pulmonary vasoconstriction, pulmonary oedema, thrombosis, and fibrosis, a focus on RAS inhibition, a key driver of (a) Pulmonary Complications - pro-thrombotic and inflammation in the lung (b) Innate Immune Response and (c) Vascular Response - vasoconstriction, inflammation and coagulation is key.

1a. Pulmonary Complications - pro-thrombotic and inflammatory lung disease. During early 2020, an enormous amount was written on the pathogenesis of COVID-19, including comprehensive summary review articles with strong emphasis on pulmonary pathophysiology in this condition (11-13, inter alia). The relationship between the dysregulation of the pulmonary renin angiotensin system (RAS) and the progression to severe acute respiratory syndrome (SARS) including the intermediate stages of infections and pneumonia has been discussed at length. By way of summary the fundamental initiation is the role of ACE2 as the receptor for SARS coronavirus infection (14). It is the impact of this initiation that drives a pulmonary dysregulation of the finely balanced RAS. This balance involves the generation of angiotensin II by ACE and the inactivation of that peptide by ACE2. The functional role of ACE2, its catalytic product angiotensin1-7 and the MAS receptor are the counterbalance moieties for angiotensin II and its AT1R receptor (11). The interaction of angiotensin 1-7 with MAS receptors promotes an anti-inflammatory response, vasodilation an anti-fibrotic response and is seen as protective. Down regulation of ACE2 in acute lung injury models with SARSCoV infection supports a protective role for RAS blockade in this form of pulmonary infection 
(11). The core hypothesis is that a dysregulated RAS is core to the pathophysiology of COVID-19 driven lung damage and acute respiratory distress syndrome (15). This builds upon much earlier experimentation showing the angiotensin II receptor blocker (ARB) losartan could effectively reduce acute lung injury induced by SARS-CoV (16).The summary statement is most apt: "Therefore, in acute lung injury, ACE, Ang II, and AT1R function as lung-injury-promoting factors, while ACE2 protects from lung injury" (17).

It follows that from a repurposing standpoint that there exists a strong target homology with agents that promote RAS inhibition and the pathogenesis of COVID-19 disease in the lung in terms of pathophysiology and pharmacology.

1b. Innate Immune Response. Angiotensin II, via activation of the AT1R receptor, is pivotal in modulating immune responses. As highlighted by Veerappan et al 2008 (18) mast cells are found in the submucosal tissue in human bronchi in the upper and lower respiratory tree. Mast cells rapidly recognise pathogens and mount an immune response that includes plasma extravasation, immunological and cytokine modulation, leukocyte recruitment and ultimately cellular inflammation (19). What is critical is the role of mast cells in the bronchi in the release of renin and the initiation of the production of the angiotensin II that acts through AT1R receptors (18). Moreover, in an experimental model of hypersensitivity, anaphylactic mast cell degranulation has been shown to result in angiotensin bronchoconstriction (18). Of significance is the observation that mast cell chymase can generate angiotensin II independently of ACE activity (20), the salient point being that ACE is membrane bound and chymase upon release moves within interstitial spaces. $\mathrm{ACE}$ also modulates macrophage and neutrophil function in its role in the innate and adaptive response (21).

Angiotensin II mediates its pro-inflammatory responses by signalling through AT1R receptor.

These responses include, leukocyte recruitment, increased production of reactive oxygen species by receptor (AT1R) activation of NADPH oxidase, contributing to inflammation and the stimulation of the innate immune responses (summarised in 21). As mentioned above angiotensin 1-7 serves as a counter to the proinflammatory influence of angiotensin II. Angiotensin 1-7 achieves this by acting through the MAS receptors expressed at the surface of the bronchial smooth muscle and alveolar epithelium (12). With the viral infection in COVID-19 the cytokine storm is a key characteristic. The release of large amounts of pro-inflammatory cytokines and chemokines (IFN $\alpha$, IFN $\gamma$, IL-1 $\beta$, IL-6, IL-12, IL-18, IL-33, TNF $\alpha$, TGF $\beta$, CXCL10, CXCL8, CXCL9, CCL2, CCL3,CCL5) by immune effector cells drives the aberrant inflammatory response (22).

Many cells of the innate immune system have been shown to have an important role in the development of human COVID lung. Pulmonary RAS has a key role in bronchoconstriction consistent with the release of renin and subsequent angiotensin II formation with mast cell degranulation (18). Moreover, in an animal model of hypersensitivity mast cell degranulation was associated with angiotensin II mediated constriction, inhibited with an AT1R blocker or an inhibitor of renin (18). From a repurposing standpoint that there exists a strong target homology with agents that promote RAS inhibition and the modulation of innate immune responses in the lung.

1c. Vascular; vasoconstriction, inflammation and coagulation.

There is growing evidence that there exists a confluence of vascular and diffuse alveolar damage with SARS and possibly also COVID-19 disease. In some of the data with COVID-19 pneumonia, McGonagle et al (23) have drawn attention to the similarities of blood vessel wall oedema, modest vessel wall immune cell infiltration, hyaline thrombosis and haemorrhagic change described earlier for patients with SARS, consistent with the observation that disseminated intravascular coagulation may develop late in COVID-19 disease. Consistent with this view Teuwen et al 2020 (24) suggested that pulmonary endothelial cells (ECs) have been largely overlooked as a target in COVID-19, as the attending ECs altered vessel barrier function promotes a pro-coagulative vascular inflammatory state and an inflammatory cell infiltration. They also highlight the fact that SARS-CoV-2 binds to the ACE2 receptor impairing the enzyme that is the counterbalance for the vasoactive angiotensin II. The lung pathology COVID-19 disease displays microvascular thrombosis and haemorrhage with extensive alveolar and interstitial inflammation termed lung-restricted vascular 
immunopathology (23). In the early stage is distinct from diffuse pulmonary intravascular coagulopathy. Cao and Li (25) also similarly highlighted the two hallmarks of critical patients with COVID-19 disease namely progressive inflammation and the trend to hypercoagulation. Furthermore, they draw attention not only to the inflammatory state as a trigger for coagulation, but endothelial cell disseminated intravascular coagulation, characterising the COVID-19 hypercoagulable state as displaying elevated D-dimer (a fibrin degradation product) and fibrinogen concentrations and prolonged prothrombin time (25).

Previous studies have shown that in pulmonary hypertension there is a reduced ACE2 activity and that augmentation of ACE2 reduced oxidant and inflammatory markers as well as improving pulmonary hemodynamics (26). Thirty years ago, our team established that in hypertensive blood vessels the endothelial cell mediated relaxation was impaired and this could be reversed with impairment of prostanoid system or treatment with the ACEI captopril (27). Collectively demonstrating a key role of the vascular endothelium in coagulation, inflammation and vasoconstriction and the capacity for abnormal function driven by the RAS. The RAS influence on the vasculature also extends to trophic factors. The neurotrophic peptide NGF is believed to be a pro inflammatory mediator in airways and promotes bronchial hyperresponsiveness, Freund-Michel et al. (28). Of significance was our demonstration that the AT1R blocking agent losartan reversed the abnormal concentrations of NGF in blood vessels in hypertension (29).

As we indicated earlier there is growing evidence that there exists a confluence of vascular and diffuse alveolar damage with SARS and possibly COVID-19. Gonzalez-Jaramillo et al 2020 in a commentary on the double burden of COVID-19, also implicates the RAS in chronic pulmonary disease such as pulmonary fibrosis and pulmonary arterial hypertension (30). Similarly, PAH is characterized by reduced ACE2 activity and the production of Angiotensin-(1-7) by ACE2 activating Mas receptors activation improves experimental models of PAH (26).

As pointed out by Cao and Li 2020 (25), SARS-CoV-2 can initially replicate in the upper respiratory track and later in the disease course replicate in the lower respiratory tract generating a secondary viremia with a subsequent attack on organ targets expressing ACE2. A dynamic consistent with the possibility outlined by Kreutz et al 2020 (11) and other groups that ACEIs and ARBs may be associated with lower incidence and/or improved outcome in patients with lower respiratory tract infections. From a repurposing standpoint that there exists a strong target homology with agents that promote RAS inhibition that involves blood vessel and vascular endothelial cell damage.

\section{Target homology of COVID-19 and therapeutic agents .}

SARS-CoV-2 is an RNA virus and they have high mutation rates up to a million times higher than their hosts, and it is this high rate that enables the viruses to avoid host immunity and to achieve drug resistance (Pachetti et al 2020- (31). From the standpoint of drug repurposing the selected drug should target, not the virus, but that aspect of the pathophysiology of COVID-19 that is tightly linked to the conserved mutational characteristics of the virus and its class.

2a. Conserved target for SARS-CoV-2. It is now generally accepted that ACE2 is the receptor for SARS-CoV and SARS-CoV-2 to enable entry to the host (32). Moreover, South et al 2020 (33) suggested that angiotensin may promote both lung injury and ARDS in SARS-CoV, SARS-CoV-2/COVID-19 and possibly in MERS.

Using crystal structures for NL63 coronavirus (NL63-CoV) and SARS coronavirus (SARS-CoV) receptor binding domains $\mathrm{Wu}$ et al 2011 demonstrated virus binding hot spots on their common receptor, the human angiotensin-converting enzyme 2 (ACE2). From that experimentation $\mathrm{Wu}$ et al 2011 (34) suggested that the hot spot features were amongst the driving force for the convergent evolution of these two viruses. It follows that this would infer multiple binding opportunities for differing or mutated viruses but restricted to a common protein target. The targeted physiological process for driving pulmonary inflammation will be hard for a virus to abandon without significant penalty and can be viewed as conserved. From a repurposing standpoint that there exists a strong target homology with agents that promote RAS inhibition and the common conserved site of the pathogenesis for COVID-19 and related viruses. 
3. Prevention to Treatment. An understanding of how the repurposed drug provides benefit in treating the host across the cascade from prevention to the life-threatening consequences of other viral pathogenesis in COVID-19 disease. Cao and Li 2020 importantly summarise the clinical course of SARS-CoV-2 infection by dividing it into three phases: viremia phase, acute phase (e.g. with pneumonia) and severe or recovery phase (25). From the standpoint of drug repurposing in COVID we have adopted a similar approach, with particular focus on the severe phase, where the host system has switched on innate immune system, affecting inflammation. oedema, and vasconstriction. Our treatment focus in this phase is on RAS inhibition and (a) predisposition and age (b) predisposition with hypertension and co-morbidities (c) treatment and secondary infection and (d) intubation.

3a. Predisposition and age. Increasing age has been shown to be a strong correlative factor with morbidity and mortality with COVID. As summarized by Shahid et al. studies have highlighted the relatively higher mortality rates in older adults (35). While a variety of reasons may underpin this predisposition, it would appear that the RAS has a strong input to aging by way of mitochondrial function, specifically a strong relationship between free radicals, the mitochondria and aging, with excessive free radical production linked to dysregulation of RAS (36). Key in this dysregulation is the angiotensin receptor AT1R and its linkage to NADPH and increased production of reactive oxygen species (ROS), and a role of RAS in decreasing ROS scavenging enzymes. As highlighted by Wilson et al 2016 (37) in mitochondria the receptors for angiotensin (AT1R and AT2R) are linked to respiration and nitric oxide production. Furthermore the presence of angiotensin 1-7 in purified mitochondria and the conversion of angiotensin I to angiotensin 1-7 by endopeptidases suggests a intramitochondrial pathway for formation of angiotensin 1-7 (37). Recently Wang et al (38) have shown that overexpression of ACE2 through mitochondrial function enhances the protective effects of endothelial progenitor cells (EPC) on endothelial cell injury. It remains to be determined whether the dysregulation of RAS described in COVID-19 also similarly has a deleterious effect on mitochondrial function, as there appears to be an emerging possibility that a complex intersection between aging, mitochondrial function, apoptosis, the RAS and coronaviruses exist. Lai et al conducted a proteome analysis on human promonocyte HL-CZ cells expressing SARS CoV3CL., finding that $36 \%$ of the up regulated proteins were located in the mitochondria, including the apoptosis-inducing factor ATP synthase beta chain and cytochrome c oxidase. They suggested that SARS CoV3CLpro could induce mitochondrial-mediated apoptosis (39). Additionally, it is possible that mitochondrial dysregulated RAS and free radical production in aging acts in concert with viral RAS dysregulation in COVID-19 disease. From the standpoint of repurposing what is important is the observation that inhibition of RAS with enalapril and losartan preserve mitochondrial function from the effects of aging (40).

3b. Predisposition and comorbidities e.g. hypertension, other vascular disease and diabetes.

Much has been written recently on the relationship between hypertension and COVID-19 disease. Patients with severe COVID-19 are often older and with a history of hypertension as seen for example in $75 \%$ of patients that died in the COVID-19 pandemic in Italy had hypertension (11).

As indicated earlier ACE2 is not only the target for SARS-CoV-2 but functionally balances the pulmonary and possibly vascular RAS/inflammation axis. Therefore, a potential increase in ACE2 mediated with antihypertensives, that act to inhibit RAAS, raised potential concerns with patients with COVID-19. There was insufficient data to determine whether these findings translated to humans and that clinical trials were underway to test these inhibitors in COVID-19 (41). Furthermore, Kreutz et al (11) indicated that after a review of the evidence there was currently no reason to discontinue RAS blockers in stable patients in the COVID-19 pandemic.

Consistent with this view there are now a growing number of studies based on medical records of hospitalized COVID patients that do not indicate a deterioration of patients with RAS inhibition and to varying extents an improvement in outcomes when compared to patients not treated with RAS inhibitors (42-45). From the preceding studies however, it is not apparent whether the RAS inhibitors were maintained during hospitalisation or possibly ceased upon admission. This may be an important consideration based upon the results of previous studies with patients with viral pneumonia. Mortensen et al (50) showed that both prior 
and continued ACEI was associated with decreased mortality and length of stay in hospitalised patients with pneumonia. Henry et al (46) determined the effects of ACEIs and ARBs in patients with viral pneumonia, finding that patients on ACEIs prior to admission and subsequently discontinued had a higher mortality than those not on ACEIs prior to admission and there was a decrease in intubation or mortality in patients with continued use of ACE inhibitors during hospital admission. These results illustrate the importance of administrative/observational data consistent with the view that observational studies based on both big and small data sets are useful in understanding how to treat viral disease (4).

What is of significance is the emerging understanding that cardiovascular disease like COVID-19. is a partially inflammatory state. In addition to the extensive discussion on hypertension and cardiovascular disease in COVID-19 there is a increasing body of evidence a role of inflammation and immunity in hypertension and cardiovascular disease (47). For example, there is a growing appreciation of the role of oxidative stress in the arterial wall, inflammatory infiltration, regulatory and interleukin 17 producing T-cells leading the development of hypertension (48).

A role for inflammation in hypertension is documented as is a powerful role of inflammation in COVID19 disease (13). It is not surprising with this confluence that hypertension and related vascular disorders enhance susceptibility to this disease. It follows that from a repurposing standpoint a growing view would be not to abandon RAS inhibitors but rather explore their potential as repurposing agents in COVID-19.

3c. Secondary pulmonary Infection.

Acute respiratory distress syndrome (ARDS) is a severe form of acute lung injury with a high mortality rate and pneumonia; infections such as COVID and sepsis are predisposing factors together with aspiration (summarised in 17). Twenty years before COVID, it was suggested that the RAS had a role in the pathogenesis of ARDS and demonstrated that angiotensin converting enzyme insertion/deletion polymorphism was associated with susceptibility and outcome in ARDS (49). This has been shown in human data with COVID, mortality disproportionally affecting Blacks who commonly have DD mutation. Even back in 2002 this team suggested that that ACE inhibitors may lower the risk of developing ARDS or reduce the severity of the disease. Subsequently in animal experiments in acute lung injury angiotensin II is upregulated and mediates acute lung failure by way of the AT1 receptor and that ACE2 and the AT2 receptor are protective against this injury (16). Consistent with theses findings Yan et al using influenza A H5N1 virus infection in mice showed that the ARB losartan improved animal survival and ameliorated acute lung injury (6).

Based on a retrospective cohort non COVID pneumonia study Mortensen et al 2012 (50) examined the influence of statins and inhibitors of the RAS on outcomes. They concluded that statins and to a lesser extent ACE inhibitors and ARBs improved these outcomes. The potential benefits of the pulmonary effects of ARBs aware seen, and he speculated that having increased ACE2 expression with pre-existing ARB treatment may be of benefit in the course of SARS-CoV-2 infection (13). In a similar fashion in a recent review on COVID-19 (11) concluded that with patients with lower respiratory tract infections ACEIs and ARBs may be associated with improved outcomes. From a repurposing standpoint the prevailing view would be that inhibitors of RAS may be of benefit in lower respiratory tract infections.

$3 \mathrm{~d}$. Intubation. Severe gas exchange problems requiring mechanical ventilation can occur with COVID19 disease. As indicated by Kim et al 2017 acute respiratory distress syndrome (ARDS) is refractory hypoxemic respiratory failure with a high mortality rates, which have been reported to range from $30 \%$ to $70 \%$ (51). Animals studies of Jerng et al 2006 have suggested a link between mechanically induced proinflammatory responses and the RAS. In particular the observation that mechanical ventilation can produce a proinflammatory response in the lungs (52). Moreover, they demonstrated that RAS is involved in the pathogenesis of the ventilator induced lung injury. Of particular importance was the observation that pre-treatment with captopril or concomitant infusion of losartan attenuated the lung injury and inflammation (52). A role of Angiotensin 1-7 in improving key responses in an experimental model of ARDS was described by Zambelli et al 2015 (53). They demonstrated that Angiotensin 1-7 reduced the severity of lung injury 
and inflammation following pulmonary acid aspiration and high stretch mechanical ventilation.

Subsequently Kim et al. (52) examined the medical records of patients admitted to a medical intensive care unit for mechanical ventilation support. They concluded that an ACE inhibitor or ARB may have beneficial effect on ARDS patients. From the standpoint of repurposing what is important is the observation that inhibition of RAS is implicated in ventilation proinflammatory response in the lungs, that COVID-19 is a disease of RAS dysregulation and that observational data with RAS inhibitors suggests they may be beneficial.

4. Dose and Safety . An application of the repurposed drug within the approved dose ranges for its initial indications for which the pharmacokinetics and toxicology are documented as part of the registration for the drug's initial indication. In particular to identify what is known about RAS inhibitors in regard to (a) observational data based upon administrative records, (b) the relationship between the existing Cmax and EC50 for new indication, (c) the historical safety of the proposed repurposed drug including knowledge of clinical maximal dose.

4a. Observational data based upon administrative records.

The key question here is not just around the evidence that the agents that inhibit the RAS improve patient outcomes in COVID-19 disease but at what doses - both for efficacy and for toxicity.

Over the last several months in 2020 we are aware of four clinical observational based studies, (42-45) all derived from medical records of patients hospitalised for COVID-19. As indicated above these studies did not indicate a deterioration of patients with RAS inhibition and conversely to varying extents an improvement in outcomes when compared to patients with COVID-19 disease not treated with RAS inhibitors. By inference these patients were likely being treated prior to hospitalisation for hypertension or diabetes on the standard drug regimens. Based on observational data there would be an argument to explore further the possibility of a new indication for RAS inhibitors as repurposed drugs in COVID-19.

4b. The relationship between the existing Cmax and EC50 for new indicatio n.

The specific question of interest here is whether there is evidence that RAS agents are effective at doses below the approved Cmax in attenuating pulmonary responses and preventing or reversing the aberrant inflammatory pathophysiology. This is also important dosing knowledge to reduce toxicity.

In a comprehensive review on repurposing (10) potential differences in dosing amounts and schedules in the area of repurposing. Our experience has indicated that if the ED50 for the new indication for the repurposed drug is equal to or less than the Cmax achieved by the drug in registered dosing schedules for its original indication then dosing issues become less complex and the arguments for registration of the repurposed drug for a new indication much easier. The point being that the bioavailability, toxicology and pharmacosurveillance of the drug at that dose, is well established as part of the original drug approval process, albeit the disease being treated is different. This latter point may introduce issues around dose-response and also dose-toxicity, but they can be surveyed from an a priori basis rather than from no knowledge.

By inference in the studies based upon observational data described immediately above (4a) the COVID-19 patients were likely being treated prior to hospitalisation for hypertension or diabetes on the standard drug regimens. If that were the case then it could be assumed that the RAS inhibitors would have been employed at or below their approved Cmax obtained from the top of the dosing range for the RAS inhibitors.

As there is no human information to date on these preclinical aspects, we need to analyse known pharmacological data from cellular systems and animals; and use supporting data from human studies of other viruses that cause ARDS in a pathophysiological process resembling COVID.

Based upon the observational data there would be an argument to explore further the possibility of a new indication for RAS inhibitors as repurposed drugs in COVID-19 initially within the existing approved Cmax of the drugs 
4c . The historical safety of the proposed repurposed drug.

We need to know the historical safety of proposed RAS inhibitors in exploring their potential role as repurposed drugs for treating COVID-19. Losartan, telmisartan, olmesartan (and additional AT1R antagonists) have been widely applied in the clinic since the 1990s for control of hypertension, diabetes and some kidney disorders, and are known as safe drugs that are rarely implicated in adverse drugs events (54-55).

Based on historical safety experience data there would be an argument to explore further the possibility of a new indication for RAS inhibitors as repurposed drugs in COVID-19.

\section{Conclusion}

The importance of the host response in infectious disease has been highlighted very recently including the suggestion that ARBs and statins, both very cheap, off patent and in our pharmacies currently could be evaluated to improve outcomes including survival in COVID-19 patients (5). This approach builds upon the pioneering earlier (4, treating of the host concept, based upon an understanding that a beneficial clinical phenotype has been observed with a treatment and this can be employed for the purpose of treating the host.

The approach brings together three concepts; namely the value of therapeutics in stabilizing patients prior to subsequent interventions, repurposing therapeutics for new indications and potential of treating the host. During this review the following became evident;

- There is a need to comprehensively determine if there exists an alignment of the proposed repurposed drug with the known pathophysiology of the viral infection. In the case of RASI what is remarkable is the interplay between an existing predisposition to RAS induced inflammation with an overlay of severe RAS dysregulation with COVID-19 disease.

- The fundamental importance of observational (administrative)data as a pathfinder source of information from which to select candidate drugs for evaluation in a repurposed context.

- The importance to adhere to the principals of repurposing as they relate to dose and safety.

We have described a framework for treating the host with repurposed drugs and illustrated its use for RAS inhibitors. We would caution that even though there exists observational data highlighting a potential protective effect of RAS inhibition consistent with a pathophysiological alignment this outcome may still be due to chance Therefore based on our analysis we believe a clinical trial for RAS inhibitors would be justified and note that eight trials of this type have already been registered.

Finally, with the advent of COVID-19 disease discussion has moved to delineating the treatment of the host from treatment and elimination of the infective agent to treating the host as out lined and pioneered by David Fedson.

\section{Competing Interests' Statement - None \\ REFERENCES}

1. Sanders J, Monogue M, Tomasz Z. Jodlowski, Cutrell J. Pharmacologic Treatments for Coronavirus Disease 2019 (COVID-19) A Review JAMA. doi:10.1001/jama.2020.6019

2. Fedson DS, Jacobson JR, Rordam OM, Opal SM. 2015. Treating the host response to Ebola virus disease with generic statins and angiotensin receptor blockers. mBio 6:e00716-15. https://doi.org/10.1128/mBio.00716-15.

3. Fedson D.S. 2016. Treating the host response to emerging virus diseases: lessons learned from sepsis, pneumonia, influenza and Ebola. Ann Transl Med 4:421. https://doi.org/10.21037/atm.2016.11.03

4. Fedson D.S.. Observational studies help us understand how to treat pandemic influenza and other emerging virus diseases J Emerg Crit Care Med 2017;1:10

5. Fedson DS, Opal SM, Rordam OM. 2020. Hiding in plain sight: an approach to treating patients with severe COVID-19 infection. mBio 11:e00398-20. https://doi.org/ 10.1128/mBio.00398-20. 
6. Yan Y, Liu Q, Li N, Du JC, Li X, Li C, Jin NY, Jiang CY. Angiotensin II receptor blocker as a novel therapy in acute lung injury induced by avian influenza A H5N1 virus infection in mouse. Sci China Life Sci, 2015, 58: 208-211, doi: 10.1007/s11427-015-4814-7.

7. Martin and Bowden. https://www.mja.com.au/journal/2020/drug-repurposing-era-covid-marketfailure-needing-leadership-and-government-investment Print June 6, 2020. Online, accessed May 30, 2020.

8. Martin JH, Clark J, Head R. Buying time: Drug repurposing to treat the host in COVID-19H Pharmacol Res Perspect. 2020;00:e00620. https://doi.org/10.1002/prp2.620

9. Pirofski L, and Casadevalli A. The Damage-Response Framework as a Tool for the Physician-Scientist to Understand the Pathogenesis of Infectious Diseases JID 2018:218 (Suppl 1): S7

10. Pushpakom S, Iorio F, Eyers PA, et al. Drug repurposing: progress, challenges and recommendations. Nat Rev Drug Discov. 2019;18(1):41-58. doi:10.1038/nrd.2018.168

11. Kreutz R, Algharably E, Azizi M, et al. Hypertension, the renin-angiotensin system, and the risk of lower respiratory tract infections and lung injury: implications for COVID-19 European Society of Hypertension COVID-19 Task Force Review of Evidence Cardiovascular Research (2020) 0, 1-12 REVIEW doi:10.1093/cvr/cvaa097

12. Verdecchiaa P, Cavallinia C, Spanevellob A, et al The pivotal link between ACE2 deficiency and SARSCoV-2 infection. European Journal of Internal Medicine, https://doi.org/10.1016/j.ejim.2020.04.037.

13. Danser J, Epstein M, Batlle D. Renin-Angiotensin System Blockers and the COVID-19 Pandemic. At Present There Is No Evidence to Abandon Renin-Angiotensin System Blockers. Hypertension. 2020;75:00-00. DOI: 10.1161/HYPERTENSIONAHA.120.15082.

14. Li W, Moore MJ, Vasilieva N, Sui J, Wong SK, Berne MA, Somasundaran M, Sullivan JL, Luzuriaga $\mathrm{K}$, Greenough TC, et al. Angiotensin-converting enzyme 2 is a functional receptor for the SARS coronavirus. Nature. 2003;426:450-454. doi:10.1038/nature02145.

15. Brandon M H, Jens V, Giuseppe L. Response to the emerging novel coronavirus outbreak. BMJ 2020;368:m406.

16. Kuba K, Imai Y, Rao S, Gao H et al. A crucial role of angiotensin converting enzyme 2 (ACE2) in SARS-coronavirus-induced lung injury. Nat Med,2005, 11: 875-879.

17. Imai Y, Kuba K and Penninger J. The discovery of angiotensin-converting enzyme 2 and its role in acute lung injury in mice Exp Physiol 2008 93.5 pp 543-548 543.

18. Veerappan A, Reid, A, Estephan R, O'Connor N, Thadani-Mulero M, Salazar-Rodriguez M, Levi R, and Silver R. Mast cell renin and a local renin-angiotensin system in the airway: Role in bronchoconstriction PNAS; January 29, 2008; vol.105(4):1315-1320.

19. Jonas S, Erjefa J. Mast cells in human airways: the culprit? Eur Respir Rev 2014; 23: 299-307. DOI: 10.1183/09059180.00005014.

20. Becker BF. All because of the mast cell: blocking the angiotensin receptor-1 should be better than inhibiting ACE (theoretically) Cardiovascular Research (2011) 92, 7-9; doi:10.1093/cvr/cvr214

21. Bernstein K, Khan Z, Giani J, Cao D-Y, Bernstein E, Shen X. Angiotensin-converting enzyme in innate and adaptive immunity Nat Rev Nephrol. 2018 May ; 14(5): 325-336. doi:10.1038/nrneph.2018.15

22. Coperchinia F, Chiovatoa L, Crocea, L, et al. The cytokine storm in COVID-19: An overview of the involvement of the chemokine/chemokine-receptor system. Cytokine and Growth Factor Reviews, https://doi.org/10.1016/j.cytogfr.2020.05.003

23. McGonagle D, O'Donnell J.S., Sharif K, Emery P, Bridgewood C. Immune mechanisms of pulmonary intravascular coagulopathy in COVID-19 pneumonia. www.thelancet.com/rheumatology Published online May 7, 2020 https://doi.org/10.1016/S2665-9913(20)30121-1.

24. Teuwen L-A, Geldhof V, Pasut A and Carmeliet P. COVID-19: the vasculature unleashed Nature Reviews/Immunology 2020https://doi.org/10.1038/s41577-020-0343-0.

25. Wei Cao and Taisheng Li Research highlight COVID-19: towards understanding of pathogenesis Cell Research (2020) 30:367-369; https://doi.org/10.1038/s41422-020-0327-4

26. Hemnes A, Rathinasabapathy A, Austin E, et al. A potential therapeutic role for Angiotensin Converting Enzyme 2 in human pulmonary arterial hypertension Eur Respir J. 2018 June ; 51(6). 
doi:10.1183/13993003.02638-2017.

27. Dyer S, Frewin D, Head R. The Influence of Chronic Captopril Treatment and Its Withdrawal on Endothelium-Dependent Relaxation. Blood Press.1992 Dec;1(4):247-53. doi: 10.3109/08037059209077670

28. Freund-Michel V, Cardoso Dos Santos M, Guignabert C, et al. Role of Nerve Growth Factor in Development and Persistence of Experimental Pulmonary Hypertension. Am J Respir Crit Care Med Vol 192, Iss 3, pp 342-355, Aug 1, 2015.

29. S. Jeffreson R. Rush C. Zettler D. B. Frewin R. J. Head THE INFLUENCE OF THE RENIN ANGIOTENSIN SYSTEM ON ABNORMAL EXPRESSION OF NERVE GROWTH FACTOR IN THE SPONTANEOUSLY HYPERTENSIVE RAT Volume22, Issue6-7July 1995 Pages 478-480 https://doi.org/10.1111/j.1440-1681.1995.tb02050.x

30. Gonzalez-Jaramillo N, Low N, Franco O. The double burden of disease of COVID-19 in cardiovascular patients: overlapping conditions could lead to overlapping treatments. European Journal of Epidemiology (2020) 35:335-337 https://doi.org/10.1007/s10654-020-

31. Pachetti M, Marini B, Benedetti F, et al. Emerging SARS-CoV-2 mutation hot spots include a novel RNA-dependent-RNA polymerase variant. J Transl Med. 2020;18(1):179. Published 2020 Apr 22. doi:10.1186/s12967-020-02344-6

32. Kai H, Kai M. Interactions of coronaviruses with ACE2, angiotensin II, and RAS inhibitors - lessons from available evidence and insights into COVID-19. Hypertens Res (2020). https://doi.org/10.1038/s41440-020-0455-8.

33. South A, Diz D, and Chappell M. PERSPECTIVES Integrative Cardiovascular Physiology and Pathophysiology COVID-19, ACE2, and the cardiovascular consequences Am J Physiol Heart Circ Physiol 318: H1084-H1090, 2020. doi:10.1152/ajpheart.00217.2020.

34. Wu K, Lang Chen L, G, et al. A Virus-Binding Hot Spot on Human Angiotensin-Converting Enzyme 2 Is Critical for Binding of Two Different Coronaviruses. J Virol. 2011 Jun; 85(11): 5331-5337. doi: 10.1128/JVI.02274-10

35. Shahid Z, Kalayanamitra R, McClafferty B et al. COVID-19 and Older Adults: What We Know J Am Geriatr Soc 68:926-929, 2020.

36. Vajapey R, Rini D, Walston J and Abadir P. The impact of age-related dysregulation of the angiotensin system on mitochondrial redox balance. Frontiers in Physiology | Mitochondrial Research November 2014; Volume5; Article439, page 2.

37. Wilson B, Nautiyal M, Tan Ya M. Evidence for a mitochondrial angiotensin-(1-7) system in the kidney. Am J Physiol Renal Physiol 310: F637-F645, 2016. First published December 23, 2015; doi:10.1152/ajprenal.00479.2015

38. Wang J, Chen S, Bihl J et al. Exosome-Mediated Transfer of ACE2 (Angiotensin-Converting Enzyme 2) from Endothelial Progenitor Cells Promotes Survival and Function of Endothelial Cell. Oxidative Medicine and Cellular Longevity Volume 2020, Article ID 4213541 https://doi.org/10.1155/2020/4213541

39. Lai C, Jou M, Huang S et al.. Proteomic analysis of up-regulated proteins in human promonocyte cells expressing severe acute respiratory syndrome coronavirus 3C-like protease Proteomics 2007, 7, $1446-1460$

40. de Cavanagh E, Piotrkowski B, Basso N et al. Enalapril and losartan attenuate mitochondrial dysfunction in aged rats The FASEB Journal express article 10.1096/fj.02-0063fje. Published online April 22,2003

41. Vaduganathan M, Vardeny O, Michel T, McMurray J, Pfeffer M, Solomon S. Renin-AngiotensinAldosterone System Inhibitors in Patients with Covid-19. N Engl J Med 382;17; April 23, 2020

42. Peng Zhang; Lihua Zhu; Jingjing Cai; et al. Association of Inpatient Use of Angiotensin Converting Enzyme Inhibitors and Angiotensin II Receptor Blockers with Mortality Among Patients With Hypertension Hospitalized With COVID-19 DOI: 10.1161/CIRCRESAHA.120.317134.

43. Mehra M, Desai S, Kuy, S. Timothy D. Henry and Amit N. Patel. Cardiovascular Disease, Drug Therapy, and Mortality in Covid-19. N Engl J Med 2020; 382:e102. DOI: 10.1056/NEJMoa2007621 
44. Meng J, Xiao G, Zhang J et al. (2020) Renin-angiotensin system inhibitors improve the clinical outcomes of COVID-19 patients with hypertension, Emerging Microbes \& Infections, 9:1, 757-760, DOI: $10.1080 / 22221751.2020 .1746200$

45. Bean D, Kraljevic Z, Searle T. ACE-inhibitors and Angiotensin-2 Receptor Blockers are not associated with severe SARS- COVID19 infection in a multi-site UK acute Hospital Trust medRxiv 2020.04.07.20056788; doi:https://doi.org/10.1101/2020.04.07.20056788

46. Henry C, Zaizafoun M, Stock E, Shamande S , Arroliga A et al. Impact of angiotensin-converting enzyme inhibitors and statins on viral pneumonia PROC (BAYL UNIV MED CENT) 2018;31(4):419423 https://doi.org/10.1080/08998280.2018.1499293.

47. David G. Harrison1, Tomasz J. Guzik, Heinrich Lob1, et al. Hypertension. 2011 February ; 57(2): 132-140. doi:10.1161/HYPERTENSIONAHA.110.163576.

48. Solak Y, Afsar B, Nosratola D. Hypertension as an autoimmune and inflammatory disease Hypertension Research volume 39, pages567-573(2016).

49. Marshall R, Webb S, Geoffrey J. Bellingan, et al; Angiotensin converting enzyme insertion/deletion polymorphism is associated with susceptibility and outcome in acute respiratory distress syndrome. Am. J. Respir. Crit. Care Med. 166, 646-650 (2002).

50. Mortensen EM, Nakashima B, Cornell J, et al. Population-based study of statins, angiotensin II receptor blockers, and angiotensin-converting enzyme inhibitors on pneumonia-related outcomes. Clin Infect Dis. 2012;55(11):1466-1473. doi:10.1093/cid/cis733.

51. Kim J, Choi S-M, Lee J, Sik Park Y et al. Effect of Renin-Angiotensin System Blockage in Patients with Acute Respiratory Distress Syndrome: A Retrospective Case Control Study Korean J Crit Care Med 2017 May 32(2):154-163 https://doi.org/10.4266/kjccm.2016.00976

52. Jerng J, Yu-Chiao Hsu, Huey-Dong $\mathrm{Wu}$, et al. Role of the renin-angiotensin system in ventilatorinduced lung injury: an in vivo study in a rat model. Thorax. 2007;62:527-535. doi: 10.1136/thx.2006.061945

53. Zambelli V, Bellani G, Roberto Borsa R et al. Angiotensin-(1-7) improves oxygenation, while reducing cellular infiltrate and fibrosis in experimental Acute Respiratory Distress Syndrome Intensive Care Medicine Experimental volume 3, Article number: 8 (2015).

54. Deppe S. , Böger R. H. , Weiss J. , \& Benndorf, R. A. (2010). Telmisartan: A review of its pharmacodynamic and pharmacokinetic properties. Expert Opinion on Drug Metabolism \& Toxicology, 6(7), 863-871. 10.1517/17425255.2010.494597

55. McIntyre M, Caffe S. E., Michalak R. A \& Reid J. L. (1997). Losartan, an orally active angiotensin (AT1) receptor antagonist: A review of its efficacy and safety in essential hypertension. Pharmacology \& Therapeutics, 74(2), 181-194. 